\title{
Armenian Mathematical Union - History and Activity
}

\author{
Yuri Movsisyan (Yerevan State University, Armenia), President of the AMU
}

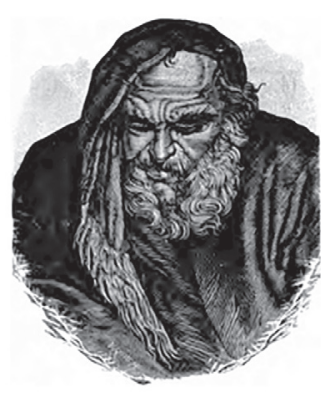

The evolution of mathematics in Armenia dates back millennia. The first indications of this were the ancient Urartian cuneiform inscriptions, particularly rich in algebraic and geometric regularities. ${ }^{1,2}$ For instance, Urartian construction workers used the golden ratio relationship between IX-VI BC before it was utilised for the facade of the Parthenon by the Greek architect and sculptor Phidias.

Interest in mathematics in Armenia grew significantly in the Early Middle Ages thanks to Anania Shirakatsi (7th c. AD), considered to be the father of exact and natural sciences in Armenia. His most valuable contribution is the textbook on Arithmetic, currently being held at the Museum of Ancient Manuscripts Matenadaran. Shirakatsi's 1400th anniversary entered the UNESCO list of important anniversaries in 2012. Other notable influencers of the time were Leo the Philosopher (9th c.), Grigor Magistros (11th c.), Hovhannes Sarkavag (11-12th cc.) and Nikoghayos Artavazd (14th c.).

In more recent history, Armenian mathematicians began forming an association during the short-lived First Republic in the year 1920. The organiser of the first mathematical association, and its first president, was a professor of Yerevan State University, Ervand Kogbetlianz.

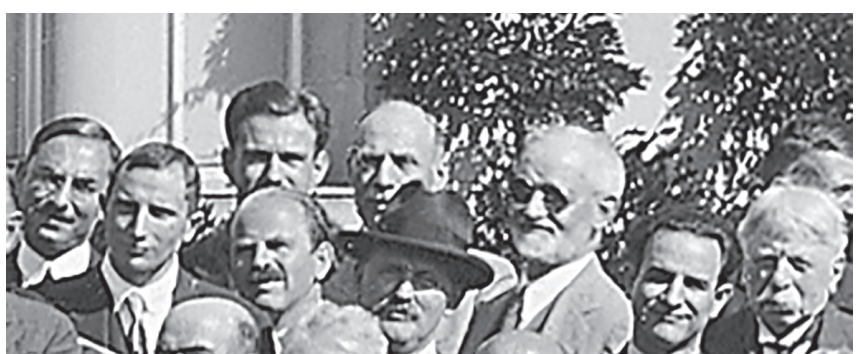

Ervand Kogbetliantz (far left) at the International Congress of Mathematicians, Zürich 1932.

Koghbetlianz's emigration to France slowed the activity of the association, until it was reinvigorated in the 1940s and 1950s by the academics Artashes Shahinyan, Mkhitar Jrbashyan and Sergey Mergelyan.

During the 1960 s to 70 s, the list of academics grew and incorporated Alexander Talalyan, Rafayel Alek-

1 Schulz, F. E. (1840). Mémoire sur le lac de Van et ses environs. JA 9: 257-323

2 Salvini, M. (2008). Corpus dei testi urartei, 1: Le iscrizioni su pietra e roccia (Documenta Asiana 8). CNR/Istituto di studi sulle civiltà dell'Egeo e del Vicino Oriente, Rome.
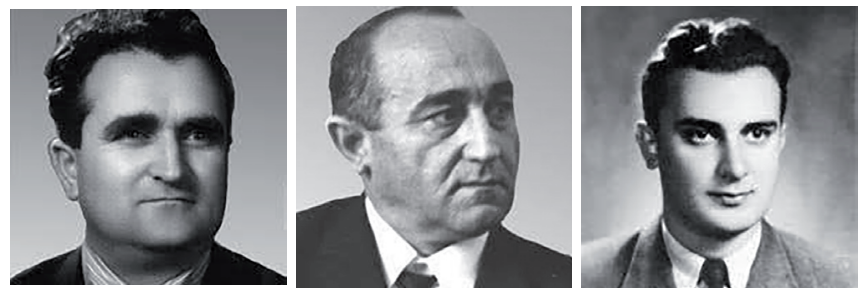

From left to right: Artashes Shahinyan, Mkhitar Jrbashyan and Sergey Mergelyan.

sandryan, Norayr Arakelian, Anry Nersessian, Ruben Ambartzumian and Rom Varshamov.
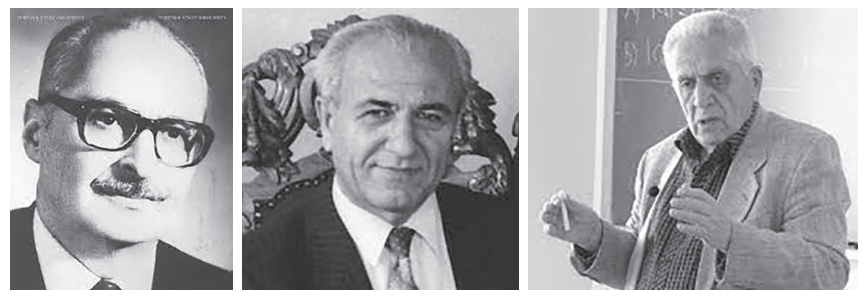

From left to right: Rafayel Aleksandryan, Norayr Arakelian and Alexander Talalyan.

The theory of functions of a complex variable, real analysis, differential equations, functional analysis and equations of mathematical physics were actively advancing in Armenia during these years. These were followed by the development of probability theory, integral geometry, algebra and mathematical logic, and discrete mathematics in the 1970 s to 80 s.

Following Armenia's independence from the Soviet Union in 1991, a group of young mathematicians formalised the association into the Armenian Mathematical Union (AMU), registering it at the Ministry of Justice with elected members of the Council and an elected president of the Union. The first elected Council was comprised of Sergey Antonyan, Samvel Apresyan, Norayr Arakelian, Victor Arzumanian, Bagrat Batikyan, Grigor Barsegian, Gegham Gevorgyan, Samvel Dalalyan, Norayr Yengibaryan, Vanik Zakaryan, Alexander Talalyan, Sargis Hakobyan, Hrachik Hayrapetyan, Tigran Harutyunyan, Felix Harutyunyan, Haik Ghazaryan, Valeriy Martirosyan, Vardan Martirosyan, Yuri Movsisyan, Karen Yagjyan, Vladimir Yavryan, Boris Nahapetyan, Romen Shahbaghyan, Faizo Shamoyan, Arthur Sahakyan, Shvayts Sahakyan, Sokrat Simonyan, Ashot Vagharshakyan and Victor Ohanyan. Many of these inaugural Council members shaped the leadership of the AMU in the years to come. The following is a complete list of the AMU's elected presidents since its formal registration: 
1991 - Alexander Talalyan

1994 - Norayr Arakelian

1997 - Ruben Ambartzumian

2001 - Norayr Yengibaryan

2004 - Hrachik Hayrapetyan

2011 - Tigran Harutunyan

2017 - Yuri Movsisyan

The mission of the Armenian Mathematical Union is to promote mathematical sciences, especially among the young generation, to establish and strengthen contacts with colleagues from around the world and with international professional organisations in the area of pure and applied mathematics and computer science. Various committees function under the AMU, the most important of them being the Education Committee, which focuses on teaching curricula, methods and the quality of textbooks.

The AMU has been a member of the International Mathematical Union since 1993 and a member of the European Mathematical Society since 2016. It is also one of the twelve members of the Silkroad Mathematics Center (SMC) that was established in 2016 under the auspices of the China Association for Science and Technology. The Union collaborates directly with a number of universities and research institutes in Armenia and abroad. It convenes general meetings, hosts discussions with visiting eminent scholars, and organises periodic thematic and general annual conferences.

The current Council of the AMU includes a number of international members, among them:

S. Adian (Steklov Mathematical Institute of RAS)

A. Arutunov (RUDN University)

S. Antonyan (Universidad Nacional Autónoma de México)

A. Basmajian (City University of New York, Hunter College)

L. Beklaryan (Central Economics and Mathematics Institute of RAS)

L. Budaghyan (University of Bergen)

S. Grigoryan (Kazan State Power Engineering University)

A. Hajian (Northeastern University, Boston)

S. Mkrtchyan (Rochester University)

R. Mnacakanov (West Virginia University)

A. Petrosyan (Purdue University)

V. Pambuccian (Arizona State University)

A. Sergeev (Steklov Mathematical Institute of RAS) H. Shahgolyan (KTH Royal Institute of Technology) M. Toomanian (University of Tabriz)

Since 2001, members of the AMU have presented the Emil Artin Junior Prize in Mathematics to former students of Armenian educational institutions for their outstanding contributions in algebra, geometry, topology, and number theory. The award is announced in the Notices of the American Mathematical Society, and carries a cash award of US\$1,000. It is named after Emil Artin, a leading 20th century mathematician of Armenian descent, and covers the fields in which Artin made major contri-

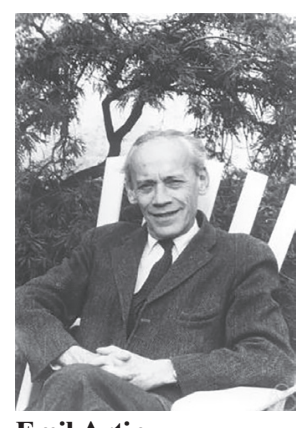

butions. The most recent 2020 recipient of the Prize was Levon Haykazyan of Oxford Asset Management. Haykazyan was chosen for his paper "Spaces of Types in Positive Model Theory", published in the Journal of Symbolic Logic in 2019.

Dedicated to Emil Artin's 120th anniversary, the AMU organised a large International Conference, celebrating his scientific heritage. The conference took place in May-June 2018 in Yerevan, Armenia, and was attended by renowned mathematicians from around the world. Concurrently, an International School in Mathematics was organised for students (mostly from around the region), featuring lectures by invited professors from the United States, Germany, Italy and the Netherlands.

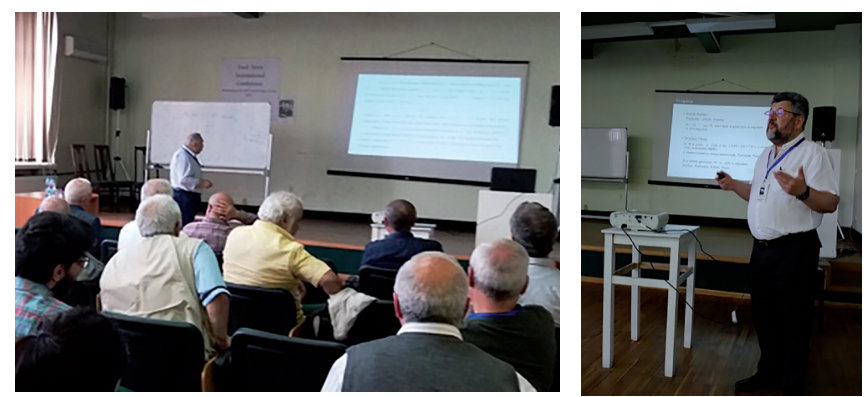

Efim Zelmanov (right) speaking at the Emil Artin International Conference in Yerevan, Armenia, 2018.

Other recent conferences organised by the AMU were: the International Conference Dedicated to the 90th Anniversary of Sergey Mergelyan (May 2018); the International Conference Dedicated to the 100th Anniversary of Mkhitar Djrbashian (October 2018); the International Conference Dedicated to the 100th Anniversary of the Yerevan State University (October 2019); and the Scientific Conference Dedicated to the International Day of Mathematics (14th March 2020).

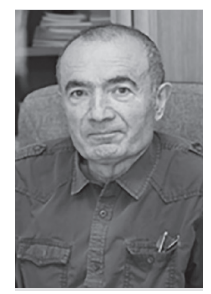

Prof. Yuri Movsisyan [movsisyan@ysu.am] is the current president of the AMU and the honorary head of the Department of Algebra and Geometry at the Yerevan State University. His research interests include algebra, mathematical logic and discrete mathematics. 